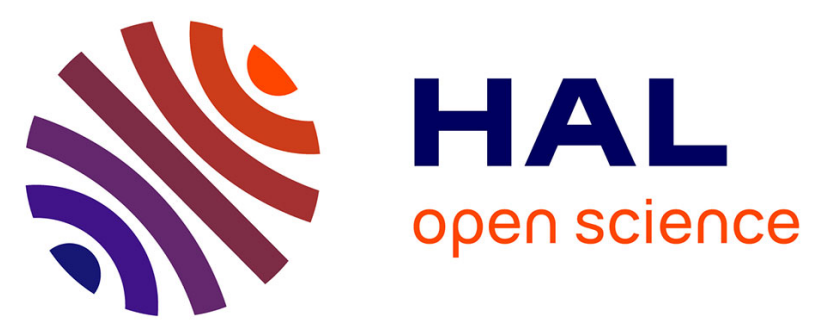

\title{
An inverse method applied to the determination of deformation energy distributions in the presence of pre-hardening stresses
}

Félix Latourte, Amine Samida, Andre Chrysochoos, Stéphane Pagano, Bertrand Wattrisse

\section{To cite this version:}

Félix Latourte, Amine Samida, Andre Chrysochoos, Stéphane Pagano, Bertrand Wattrisse. An inverse method applied to the determination of deformation energy distributions in the presence of pre-hardening stresses. Journal of Strain Analysis for Engineering Design, 2008, 43, pp.705-717. 10.1243/03093247JSA428 . hal-00514561

\section{HAL Id: hal-00514561 \\ https://hal.science/hal-00514561}

Submitted on 24 Sep 2021

HAL is a multi-disciplinary open access archive for the deposit and dissemination of scientific research documents, whether they are published or not. The documents may come from teaching and research institutions in France or abroad, or from public or private research centers.
L'archive ouverte pluridisciplinaire HAL, est destinée au dépôt et à la diffusion de documents scientifiques de niveau recherche, publiés ou non, émanant des établissements d'enseignement et de recherche français ou étrangers, des laboratoires publics ou privés. 


\title{
An inverse method applied to the determination of deformation energy distributions in the presence of pre- hardening stresses
}

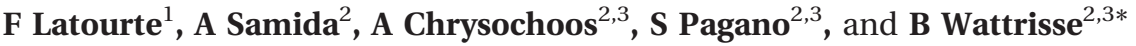 \\ ${ }^{1}$ Dynamic Inelasticity Laboratory, Department of Mechanical Engineering, Northwestern University, Evanston, Illinois, \\ USA \\ ${ }^{2}$ Mechanics and Civil Engineering Laboratory, Montpellier II University, Montpellier, France \\ ${ }^{3}$ Laboratoire de Micromécanique et d'Intégrité des Structures, Saint-Paul-lez-Durance, France
}

\begin{abstract}
This paper presents an experimental procedure to estimate the deformation energy distribution within plane samples submitted to mechanical loading. This procedure combined a digital image correlation (DIC) technique giving in-plane displacement fields with an identification method that separately provided fields of material properties and stress distributions developed during the loading. The method was first applied to simulated data to characterize the capabilities of the image processing. Finite element computations were first performed on a complex structure using a standard linear kinematical hardening model to generate multistage loadings leading to heterogeneous displacements and distributions of deformation energy. Loads and displacements were then used as inputs to check the robustness of the image processing by comparing the identified deformation energy fields with the computed ones. The procedure was then applied to experimental data. Tests were conducted under conditions similar to the numerical tests. The identification of a linear kinematical hardening model gave deformation energy patterns showing a good agreement with the simulated results, even in the presence of residual stresses induced by a prehardening.
\end{abstract}

Keywords: digital image correlation, deformation energy distributions, pre-hardening stresses

\section{INTRODUCTION}

The conversion of mechanical energy into heat has been investigated for a wide range of materials by many researchers, including those in references [1] to [5]. Using different experimental techniques (calorimeter [6], thermocouples [7], infrared (IR) sensors $[\mathbf{8}, \mathbf{9}])$, these studies gave similar results, showing that a variable amount of mechanical energy is converted into heat during inelastic transformation. Such methods generally provide a macroscopic estimate of the Taylor-Quinney co-

*Corresponding author: LMGC - CNRS UMR 5508, Universite Montpellier II, cc 048 - Place E. Bataillon, Montpellier Cedex 5, 34095, France.email: wattrisse@lmgc.univ-montp2.fr efficient that links mechanical and dissipated energies. Here, 'macroscopic' refers to the scale of the sample gauge part. Nevertheless, in the case of heterogeneous loading (e.g. necking, shear bands), global energy measurements are representative both of the material behaviour and combined structural effects. Under such conditions, the overall TaylorQuinney coefficient can no longer be representative of the sole material behaviour. The material behavioural analysis then needs a local construction of the energy balance that requires an estimate of the deformation energy locally given to the material.

In this paper, a specific procedure is presented that is designed to estimate locally the deformation energy in the case of quasi-static, heterogeneous tests during elastoplastic transformations. Various 
causes can explain the response heterogeneity of a sample, like structural effects associated with the overall loading or with the boundary conditions, or material effects resulting, for example, from a nonhomogeneous initial state of the material. The latter situation is often encountered in particular mechanical situations, such as fatigue, for which the initial state plays a key role in the subsequent material evolution. The aim of this paper is to investigate such heterogeneous responses in order to verify the abilities of the developed protocol in order to distinguish structural and material effects better in terms of deformation energy.

Deformation energy constitutes the energy input provided during a mechanical test, and its assessment is necessary to perform a local energy balance.

\subsection{DIC}

The in-plane components of the displacement vector were obtained by a DIC algorithm applied to the images recorded by a high-resolution camera (ATMEL Camelia 8M). The camera was located perpendicularly to the tested specimen fixed between the grips of a hydraulic testing machine (MTS). The average spot size of the speckle image recorded by the camera was estimated as the mean radius of the autocorrelation peak at its half height. In this case it was approximately 1 pixel.

The components of the displacement vector $\boldsymbol{u}\left(u_{x}\right.$, $u_{y}$ ) were determined at a given point $\mathrm{M}(x, y)$ whose coordinates were given with respect to the frame of reference, by maximizing the normalized correlation coefficient defined for continuous signals by

$$
C(\boldsymbol{\Phi})=\frac{\int_{\mathrm{CZ}} I_{1}(x, y) I_{2}\left[x+\Phi_{x}(x, y), y+\Phi_{y}(x, y)\right] \mathrm{d} x \mathrm{~d} y}{\sqrt{\int_{\mathrm{CZ}} I_{1}(x, y)^{2} \mathrm{~d} x \mathrm{~d} y} \sqrt{\int_{\mathrm{CZ}} I_{2}\left[x+\Phi_{x}(x, y), y+\Phi_{y}(x, y)\right]^{2} \mathrm{~d} x \mathrm{~d} y}}
$$

The set-up combined an identification method providing stress and mechanical parameter fields with an imaging technique, namely digital image correlation (DIC), now commonly used to measure displacement and strain fields on the surface of thin specimens [10].

In what follows, first the procedure allowing the estimation of the deformation energy fields associated with a heterogeneous material response is presented. Then, the method is tested on numerical examples to assess the performances and robustness of the method. Finally the identification results obtained on a dual-phase steel sample (commercial grade DP600) are discussed.

\section{DEFORMATION ENERGY ESTIMATE}

The local estimate of deformation energy requires stress and strain fields to be computed. These were obtained through a mixed approach combining experimental measurements and a numerical identification method. The strain distributions were derived from displacement fields obtained by a digital image correlation technique, while stresses were computed through the constitutive equations of an elastoplastic model previously identified on the basis of the displacement fields. In this work a J2 plasticity criterion associated with a usual linear kinematical hardening was chosen to describe the hardening effects in tension. where $I_{1}$ stands for the reference initial image and $I_{2}$ for the deformed image, $\boldsymbol{\Phi}=\left(\Phi_{\mathrm{x}}(x, y), \Phi_{\mathrm{y}}(x, y)\right)$ expresses for the local transformation between the two images, and CZ refers to the correlation zone. The local transformation can be associated, for example, with rigid-body translations [11], affine transformations [12], or quadratic transformations [13]. Here the following polynomial transformation is identified

$$
\begin{aligned}
& \Phi_{x}(x, y)=\sum_{i=0}^{d_{i}} \sum_{j=0}^{d_{j}} a_{i j} x^{i} y^{j} \\
& \Phi_{y}(x, y)=\sum_{i=0}^{d_{i}} \sum_{j=0}^{d_{j}} b_{i j} x^{i} y^{j}
\end{aligned}
$$

where $d_{i}$ and $d_{j}$ are the degrees of the polynomial transformation along the $x$ and $y$ directions respectively. The correlation computation consisted of determining the set of transformation coefficients $\left(a_{i j}, b_{i j}\right)$ that maximize the correlation coefficient $C(\Phi)$. The dedicated optimization was performed iteratively: at the first iteration, the transformation was supposed to be null, whereas for the $i$ th iteration, the in-plane displacement components $\left(u_{x}^{k}, u_{y}^{k}\right)$ of a set of points $\mathrm{M}^{k}$ located in the vicinity of the point $\mathrm{M}$ were computed as

$$
\left(u_{x}^{k}, u_{y}^{k}\right)=\underset{\left(a_{00}^{k}, b_{00}^{k}\right)}{\arg \max }\left[\widetilde{C}\left(a_{00}^{k}, b_{00}^{k}\right)\right]
$$


where

$$
\widetilde{C}\left(a_{00}^{k}, b_{00}^{k}\right)=\frac{\int_{\mathrm{CZ}} I_{1}(x, y) I_{2}\left[x+\Phi_{x}^{i-1}(x, y)+a_{00}^{k}, y+\Phi_{y}^{i-1}(x, y)+b_{00}^{k}\right] \mathrm{d} x \mathrm{~d} y}{\sqrt{\int_{\mathrm{CZ}} I_{1}(x, y)^{2} \mathrm{~d} x \mathrm{~d} y} \sqrt{\int_{\mathrm{CZ}} I_{2}\left[x+\Phi_{x}^{i-1}(x, y)+a_{00}^{k}, y+\Phi_{y}^{i-1}(x, y)+b_{00}^{k}\right]^{2} \mathrm{~d} x \mathrm{~d} y}}
$$

Here a so-called classical normalized correlation coefficient was maximized, which was associated with a rigid-body translation motion between the initial image $I_{1}$ and the final image modified by the transformation computed at the previous iteration $I_{2}\left[x+\Phi_{x}^{i-1}(x, y), y+\Phi_{y}^{i-1}(x, y)\right]$.

The maximization of $\widetilde{C}$ was performed using the discrete values of $\widetilde{C}\left(\delta x_{m}, \delta y_{n}\right)$ computed from the multiple pixel virtual displacements $\left(\delta x_{m}, \delta y_{n}\right)$. These discrete data were interpolated in the vicinity of their maximum by quadratic functions giving a subpixel displacement resolution [14].

The computed displacement components $\left(u_{x}^{k}, u_{y}^{k}\right)$ were approximated in the least-squares sense by polynomial expressions given in equation (2) to give the value of the local transformation coefficients at the current $i$ th iteration $\left[\Phi_{x}^{i}(x, y), \Phi_{y}^{i}(x, y)\right]$. This computation was repeated until convergence, and the convergence criterion was stated as the difference between the displacements computed at point $\mathrm{M}$ for two successive iterations.

For the applications presented in this paper, a biquadratic local transformation was chosen (i.e. $d_{i}=d_{j}=2$ ), and the convergence criterion was equal to 0.01 pixel. This relatively high value was chosen for all the computations (associated with both simulated and real images) in order to limit possible numerical oscillations in the iterative computation of the 18 parameters of the local transformation $\left(a_{i j}, b_{i j}\right)$. Furthermore, the quantity $I_{2}\left[x+\Phi_{x}^{i-1}(x, y), y+\Phi_{y}^{i-1}(x, y)\right]$ was estimated using a bilinear interpolation of the grey level intensity in image $I_{2}$.

The strain field was deduced by applying spatial differential operators on the displacement data. To improve the signal-to-noise ratio, the displacement field was filtered before being passed to the identification procedure. The chosen method for this operation was based on local least-squares fitting of the displacement data. The displacement field was locally approximated in the neighbourhood of each material point $M$ (i.e. a material surface element) by a given function whose gradients can also be injected in a strain computation. Naturally, both the shape of the approximation function and the size of the approximation zone (AZ) may affect the efficiency of the filtering. A choose was made here to use biquadratic approximation functions in order to ensure the consistency with the correlation formulation. The errors on strains were estimated on computer-generated speckle images whose imposed deformation pattern was consistent with the one expected for the most strained state using the methodology described in reference [11]. These images were generated in order to present characteristics similar to the ones measured on real images (in terms of histograms, correlation radii, etc.). Furthermore, the acquisition noise was simulated on these images by adding a random distribution of grey levels whose amplitude was 5 per cent of the digitalization scale. The order of magnitude of the peak-to-peak error on the strain computed with the approximation functions was $10^{-3}$, as shown in Fig. 1. The residual repartition plotted in this figure illustrates the efficiency of the filtering operation and particularly the absence of introduced bias by the filtering.

As will be presented in subsection 2.2.2, the strain field used in the identification procedure was not computed in this way, but directly deduced from the filtered nodal displacements and from the differentiation of the shape functions associated with the finite element description of the displacement field.

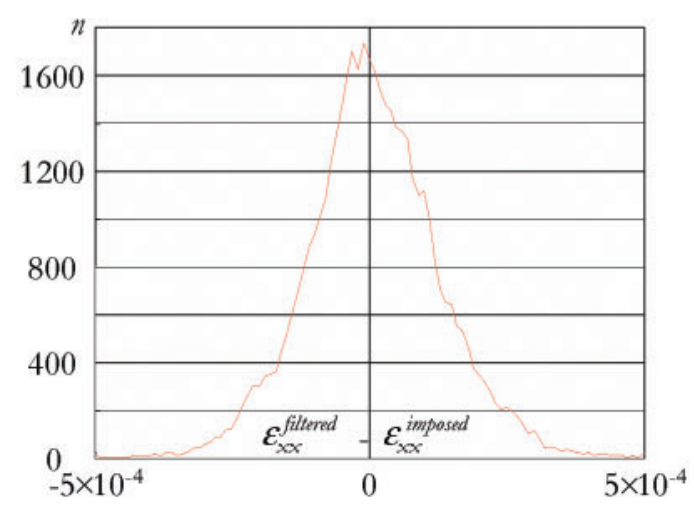

Fig. 1 Residual on the strain field obtained by fitting the displacement field histogram of $\varepsilon_{x x}^{\text {filtered }}-\varepsilon_{x x}^{\text {imposed }}$ for a simulated image, $x$ being the loading axis 
An important error source associated with DIC computations can also come from optical deformations of the recorded images due to out-of-plane movements or geometrical distortions. To limit the parasitic motions, the specimens were placed between grips that were rigidly mounted on the testing machine. The alignment was checked using the specific procedure described in reference [11]. The optical distortions were estimated using a parametric distortion model whose coefficients were identified on measured displacement fields corresponding to rigid-body motions. After being identified, the distortions were suppressed from all acquired images by applying the inverse transformation.

\subsection{Identification procedure}

The material behaviour was locally identified from in-plane displacement distributions obtained by DIC. The identification of material properties from field measurements is a particularly active research area that has led to the development of various approaches, with some of the most widespread being the finite element model updating method [15], the virtual field method [16], and the equilibrium gap method [17].

The framework chosen to state and solve the identification problem associated with an elastoplastic behaviour with positive linear kinematical hardening under the small strain hypothesis is now introduced.

\subsubsection{Variational method}

DIC provided in-plane displacement fields that gave, after derivation, fields of small strain $\varepsilon$. In the most general case, these surface measurements do not allow identification of material properties on a whole volume. For this reason, work focused on thin flat samples for which the plane stress assumption was realistic. For each deformation increment, local measurements of three in-plane strain components allowed three material parameters at most to be determined locally $[\mathbf{1 8}, \mathbf{1 9}]$.

Here the identification of a linear kinematical hardening model under the small strain hypothesis was considered [20]. An additive decomposition of the strain tensor into elastic and plastic parts was assumed. The chosen plane stress J2 plasticity model [21] depended on two kinds of mechanical parameters: first, the two independent coefficients of the isotropic elastic behaviour (Young's modulus $E$ and
Poisson's ratio $v$ ) and then the two plastic parameters (yield stress $\sigma_{0}$ and hardening modulus $k$ ). To identify this set of four parameters, a two-step method allowed first the two elastic coefficients in a pure elastic loading increment to be identified. The couple $\left(\sigma_{0}, k\right)$ was then identified on the next elastoplastic loading increments.

In the following, the elastic identification step is first presented, followed by the elastoplastic one.

(a) Identification of the elastic constants. A variational method associated with an elastic energy functional defined in reference [22] was used both to identify the field of elastic parameters and to determine the stress $\sigma$ locally. The energy functional $F$ on an appropriate domain of interest $\Omega$ (typically the sample gauge part) reads

$$
F(\tau, B)=\frac{1}{2} \int_{\Omega}(\varepsilon(u)-B: \tau): B^{-1}:(\varepsilon(u)-B: \tau) \mathrm{d} \Omega(4)
$$

where the stress field $\tau$ is statically admissible and the compliance tensor $B$ is supposed to be symmetrical, non-negative, and definite.

The functional $F(\tau, B)$ is separately convex, positive, and null at point $\left(\sigma, A_{\mathrm{e}}\right)$ if and only if $\left(\sigma, A_{\mathrm{e}}\right)$ satisfies the constitutive equation. Therefore, the identification was performed by numerically minimizing the functional $F(\tau, B)$. A minimization conducted by a relaxation method gave the solution stress field and the field of elastic parameters respectively.

During the tests the overall forces applied at the specimen boundary were recorded and passed to the identification procedure. An augmented formulation of equation (4) involving Lagrange multipliers was used to solve the static admissibility: the stress solution was compatible with the overall forces applied on some boundaries of $\Omega$ and also the stress-free boundaries.

For each loading step the variational approach provided a couple $\left(\sigma, A_{\mathrm{e}}\right)$, where $\sigma$ was a stress tensor and $A_{\mathrm{e}}$ a compliance tensor. This couple became a candidate solution to the elastic identification problem if it satisfied the comparison procedure on the local compliance tensor (i.e. the relative difference of two tensors $A_{\mathrm{e}}$ determined between two consecutive loading steps is small enough). Otherwise the problem was assumed to be plastic, and an elastoplastic algorithm allowed the hardening modulus $k$ and the yield stress $\sigma_{0}$ to be identified separately.

(b) Identification of elastoplastic parameters. In such a case, a variational method based on two energy functionals was associated with two different descrip- 
tions of the mechanical problem [20]: one based on the total displacement field, which is referred to here as 'standard formulation' and another one referred to as 'incremental formulation', based on an incremental displacement field. These formulations involved the secant and tangent plastic tensors $\left(A^{\mathrm{s}}\right.$ and $\left.A^{\mathrm{t}}\right)$ associated with the chosen model both depending on the unknown plastic parameters and the already determined elastic ones. The energy functionals considered hereafter were the previously defined functional $F$ associated with the 'standard formulation' and the functional $G$ related to the 'incremental formulation'

$$
\begin{aligned}
G(\Delta \tau, B)= & \frac{1}{2} \int_{\Omega}[\varepsilon(\Delta u)-B: \Delta \tau]: B^{-1} \\
& :[\varepsilon(\Delta u)-B: \Delta \tau] \mathrm{d} \Omega
\end{aligned}
$$

where $\Delta \tau$ represents the incremental stress field and $\varepsilon(\Delta u)$ is the strain related to the incremental measured displacement. Functionals $F$ and $G$ correspond to constitutive error gaps written in a purely mechanical framework. They do not take the irreversibility induced by plasticity into account. Nevertheless, the plastic dissipation, included in the energy balance, can be estimated via the model and the identified parameters. Using $F, G$, and two successive plastic steps it became possible to find the incremental stress field and the plastic coefficients $k$ and $\sigma_{0}$. More precisely, a fixedpoint algorithm was used to solve this problem: the functional (respectively $F$ or $G$ ) was successively minimized on $(\Delta \sigma, k)$ at fixed $\sigma_{0}$ and then minimized on $(\Delta \sigma, k)$, with $k$ being fixed at its last identified value (obtained at the previous minimization step). To enforce the positive definitiveness of tensor $B$, the hardening modulus $k$ was supposed to be positive. This hypothesis naturally excludes the investigation of softening behaviours. However, it was considered that this limitation did not affect the generality of the proposed procedure, the specimen softening being able to be interpreted as a structural effect associated with the development of strain localization, as shown in previous work [23].

\subsubsection{Numerical implementation and estimation of the deformation energy}

From a purely numerical point of view, appropriate descriptions of the stress field, the material properties, and the displacement field in each finite element were required. To carry out the direct calculation, a formulation based on a naturally equilibrated Airy function was chosen. On each mesh element, the chosen Airy potential was a third-degree polynomial.
The material properties were considered to be constant within each finite element of the mesh. One possibility was to combine element subassemblies with constant material properties. To conclude with the finite element description, it was necessary to define the displacement interpolation. In this approach, the Q2 9 element, constituted of nine displacement nodes, was chosen, as described in references [24] and [20]. The associated strain was quadratic, allowing the quadratic stress $B: \varepsilon(u)$ to remain close to the stress given by the Airy function.

By the previous identification procedure, knowledge at each loading step of both the incremental and total stress fields was assured. Furthermore, the quadratic strain associated with the measured inplane displacement could be computed in each finite element. The averaged deformation energy $w_{\text {def }}\left(\Omega_{i}, t\right)$ on a given stress element $\Omega_{i}$ at time $t$ is defined by

$$
\begin{aligned}
w_{\mathrm{def}}\left(\Omega_{i}, t\right)= & \frac{1}{\Omega_{\mathrm{i}}} \int_{t=0}^{t} \int_{(x, y) \in \Omega_{\mathrm{i}}} \sigma(x, y, t) \\
& : \dot{\varepsilon}(x, y, t) \mathrm{d} x \mathrm{~d} y \mathrm{~d} t
\end{aligned}
$$

From a numerical point of view, the following discrete form of equation (6) was used

$$
\begin{aligned}
w_{\mathrm{def}}\left(\Omega_{i}, t_{n}\right)= & \frac{1}{2} \frac{1}{\Omega_{i}} \sum_{i=1}^{n-1} \int_{(x, y) \in \Omega_{\mathrm{i}}}\left[\sigma\left(x, y, t_{i+1}\right)+\sigma\left(x, y, t_{i}\right)\right] \\
& :\left[\varepsilon\left(x, y, t_{i+1}\right)-\varepsilon\left(x, y, t_{i}\right)\right] \mathrm{d} x \mathrm{~d} y
\end{aligned}
$$

where the spatial integration is exact, using the shape functions of the finite elements, and $n$ stands for the number of load steps used for the identification.

\section{APPLICATION EXAMPLE}

The distribution of deformation energy was computed with the strain measurement fields and the computed stress fields derived from the identified model. For elastoplastic materials, the stress developed in a given situation directly depends upon the initial selfequilibrated residual stress pattern preceding the loading. Unfortunately, in most situations, this residual stress state cannot be easily determined.

For material behaviours like linear kinematical hardening, the presence of an initial residual stress state is directly translated as an offset of the current yield stress. The latter being one of the material parameters, it was then possible to account for the 
unknown initial state distribution using the identification procedure described in the previous section; the existence of a residual stress pattern was consequently translated as a distribution of yield stresses.

The aim of the proposed method was to determine the distribution of the deformation energy within a heterogeneously strained zone. Work first focused on an academic situation where the strain localization was related to a non-zero residual stress state and generated in such a way that the associated stresses were sufficiently important to be experimentally revealed. The main idea was to validate the approach on simple tests where heterogeneities could be easily tracked.

Here, the residual stress state resulted from a mechanical loading on a complex shape sample machined in an initially homogeneous material. The structural effects induced a heterogeneous hardening state of the material that generated a controlled and identifiable residual stress state after unloading. This final state represented the heterogeneous reference state for the subsequent loading step on which the deformation energy had to be estimated.

\subsection{Numerical experiment}

In a first step, the whole procedure was assessed on numerically obtained data in order to estimate its performance on 'perfect', noise-free data. By 'per- fect', it was assumed that the finite element description of the material behaviour was in complete agreement with the hypotheses conceded in the identification procedure: small perturbation hypothesis, plane stress assumption, and constitutive equations (isotropic linear elasticity and linear kinematic hardening).

\subsubsection{General description of the simulations}

The loading of the sample was simulated using a finite element code. The specimen geometry resembled the typical 'dog-bone' tensile specimen but with a trapezoidal area of additional width in the specimen centre, allowing the response of the specimen to be non-homogeneous. The loading was applied in four consecutive loading steps (see Fig. 2). The first two steps corresponded respectively to the loading and the total overall unloading of the sample, leading to the development of a heterogeneous inelastic stress state within the structure. The third step was related to the redistribution of the residual stresses associated with the 'machining' of the two triangular-shaped domains. This operation was intended to obtain the geometry of a standard tensile specimen, with a heterogeneous residual stress pattern. Numerically, this operation was done by changing the mechanical properties of the element set corresponding to these two zones and

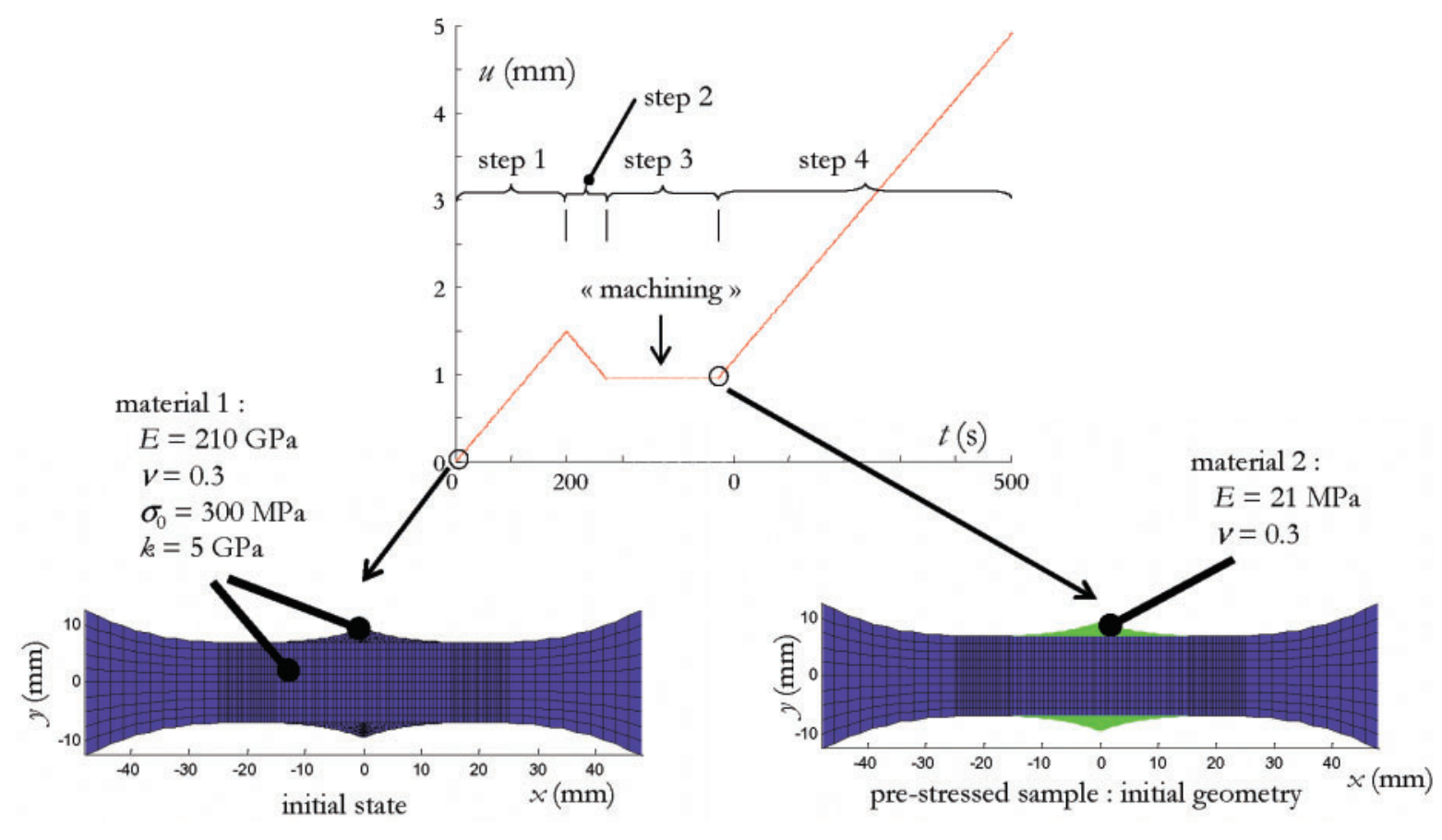

Fig. 2 Mesh and pre-deformed state of the sample 
computing the resulting self-equilibrated stress state (see Fig. 2, material 2); the rigidity of the material was divided by a factor $10^{4}$. Consequently, the mechanical contribution of these zones was negligible in terms of overall deformation energy. This operation allowed account to be taken of the nonuniform initial distribution of the hardening state variables, without any numerical difficulty. Finally, after the last simulated loading step, strain localization effects due to the heterogeneous pre-hardening state of the material were observed as expected.

\subsubsection{Residual stress state}

It has already been mentioned that the first three steps were only designed to obtain a heterogeneous residual stress state. At the end of the third loading step, the numerical simulation gave access to plastic strain and stress components. Figure 3 shows the distribution of both equivalent plastic strain and von Mises stress at this particular state. The strong similarity between the Figs 3(a) and (b) is explained by the fact that the initial state of the material (at the beginning of the first loading step) was homogeneous (i.e. $p=0$ and $\sigma_{0}=300 \mathrm{MPa}$ ).

The stress, strain, and deformation energy fields were computed with respect to this reference state. In order to illustrate the results associated with this pre-hardening state, in Fig. 4 the distribution of the axial stress and that of the deformation energy density (expressed in MPa, which is equivalent to $10^{-3} \mathrm{~J} / \mathrm{mm}^{3}$ ) given by the direct FE computation at

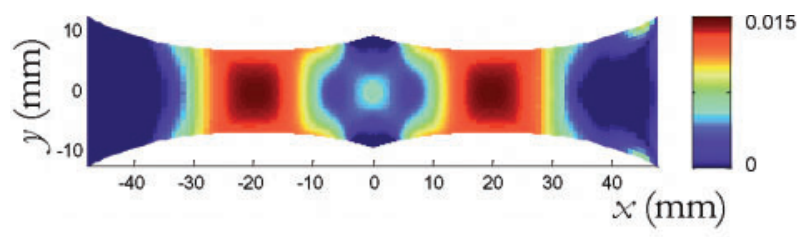

(a) equivalent plastic strain $p$ the end of the fourth step were plotted. As expected, the incremental stress and deformation energy density developed in the two triangular-shaped zones were negligible, which is consistent with their negligible contribution to the overall stress pattern. The distributions observed in Fig. 4 are not completely symmetrical due to the boundary conditions simulating the grips: uniform pressure on one side and null displacement on the other.

\subsubsection{Identification results}

The overall forces coming from the numerical simulations were directly transferred to the identification procedure described in section 2 . The inplane displacement field was interpolated, using the finite element (FE) shape functions, on a regular $27 \times 27$ grid. Using this procedure, the mechanical properties $\left(E, v, k, \sigma_{0}\right)$ and the stress field were identified on $13 \times 13$ elements.

Not surprisingly, the elastic property distributions were quite homogeneous and the mean values of the Young's modulus and of the Poisson ratio were equal to the imposed values. The local fluctuations of these identified data were associated with a standard deviation of $0.5 \mathrm{MPa}$ and $10^{-3}$ respectively. The identified plastic parameter distributions are shown in Fig. 5. It can be observed that the hardening modulus $k$ was slightly overestimated (around 3 per cent) and that its spatial fluctuation corresponded to a standard deviation of $230 \mathrm{MPa}$, which represented 5 per cent of the imposed value (see Figs 5(a) and (b)).

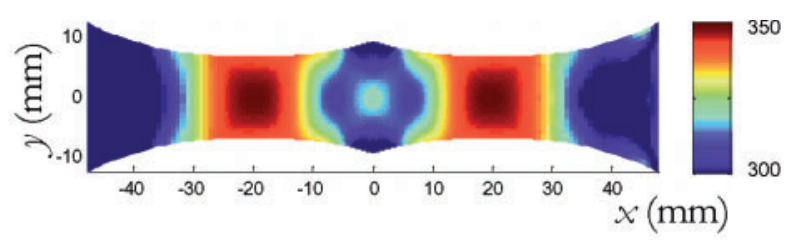

(b) current yield stress $\sigma_{0}(\mathrm{MPa})$

Fig. 3 Mechanical fields at the end of the third load step

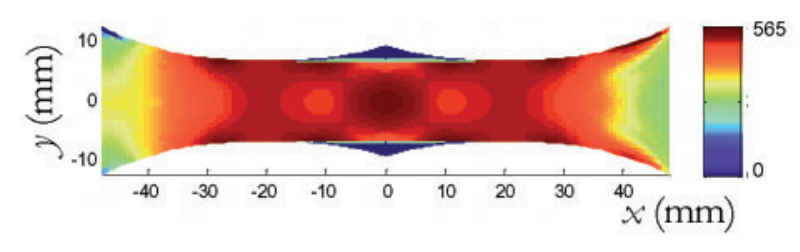

(a) axial stress $\sigma_{s \infty}(\mathrm{MPa})$

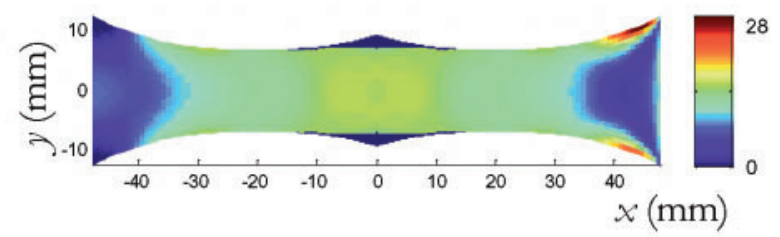

(b) deformation energy density $w_{d f}(\mathrm{MPa})$

Fig. 4 Incremental mechanical fields at the end of the fourth load step 


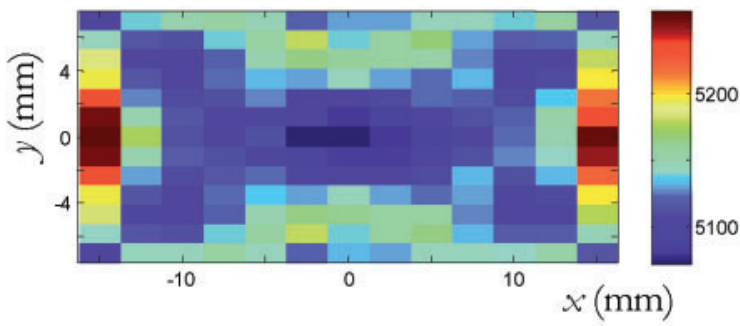

(a) $k(\mathrm{MPa})$

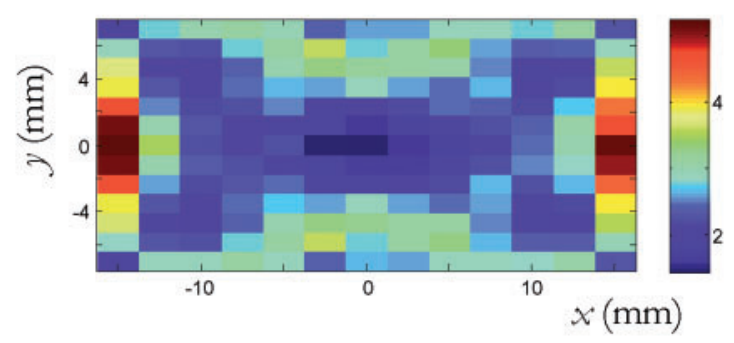

(c) relative uncertainty on $k(\%)$

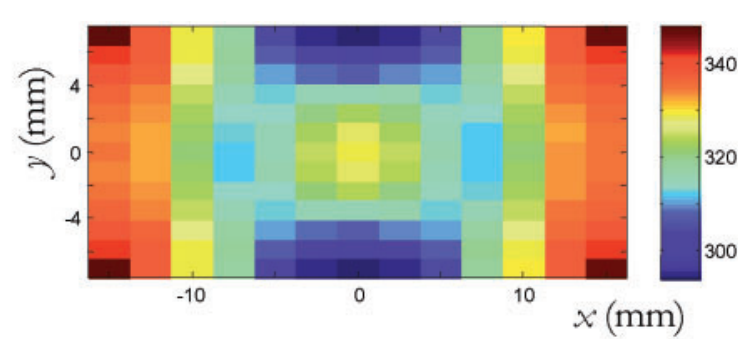

(b) $\sigma_{0}(\mathrm{MPa})$

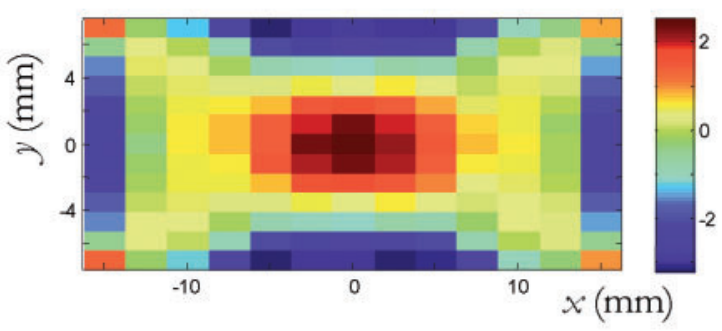

(d) relative uncertainty on $\sigma_{0}(\%)$

Fig. 5 Identified plastic parameters

The identified yield stress distribution is plotted in Fig. 5(b). The comparison between Fig. 3(b) and Fig. 5(b) shows a high correlation level between the reference and identified distributions. The identified values were quantitatively consistent with the reference ones: the maximum error of about 3 per cent was located in the zones where stress gradients were the most important (in the four corners of the domain and in the middle of the free boundary). This repartition may be due to the stress elements (see section 2) that could not account perfectly for the spatial evolution of the stress. The slight uncertainties on the stress estimates affected the computing of the yield stress, and consequently the tangent modulus.

The identified quantities directly involved in the estimation of the deformation energy are plotted in Fig. 6. As already mentioned, they depended on the identified elastic parameters and thus integrated the discrepancy on these parameters. In particular, the axial component of the identified stress tensor is shown in Fig. 6(a). It also reveals strong stress gradients in the four corners of the domain and in the neighbourhood of the two free boundaries. The identified stress pattern was consistent with the reference one. Nevertheless, the important stress gradients were slightly underestimated with a maximum difference amplitude of around 10 per cent (see Fig. 6(c)). Here it is reiterated that this quantity was determined without any a priori knowledge of the prehardening state of the material.
The deformation energy density patterns are plotted in Fig. 6(b). The displacement field used for the identification was different from the one given by the finite element computation because it had been interpolated on a much coarser grid. Consequently, the shape functions used for the deformation computation within the identification procedure were also unsuited to perfectly represent the strain gradients. These results show, however, a good consistency with the reference values. It can be noticed that the profile of the discrepancy map of the axial stress fits with that of the deformation energy (see Figs 6(c) and (d)).

The specific influence of the superimposed noise on the displacement measurements on the identification results have already been presented in reference [20]. It was observed that the noise did not affect the systematic patterns of the identification errors, but induced a supplementary random error distribution whose variance increased quasilinearly with its amplitude.

\subsection{Real experiment}

A similar experiment was carried out on a specimen machined in a $2.5 \mathrm{~mm}$ thick laminated sheet of DP600 steel. The initial geometry of the sample and the loading sequences were identical to the ones used in numerical simulations. The first loading step 


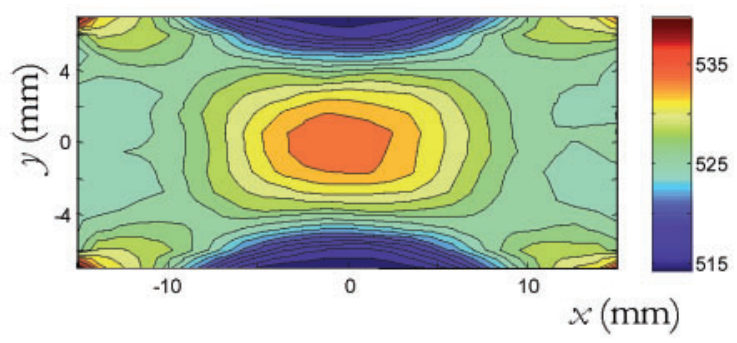

(a) axial stress $\sigma_{\infty \infty}(\mathrm{MPa})$

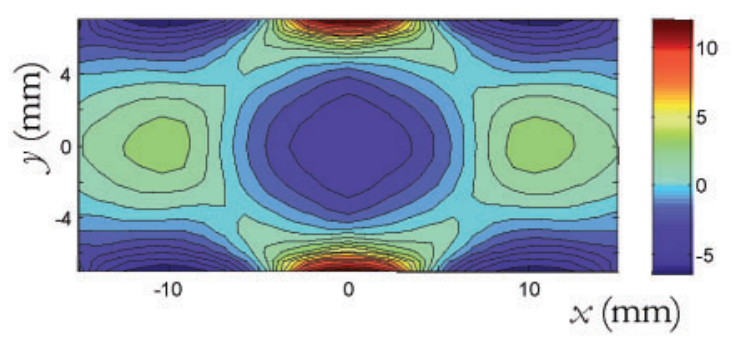

(c) relative uncertainty on the axial stress $\sigma_{\infty x}(\%)$

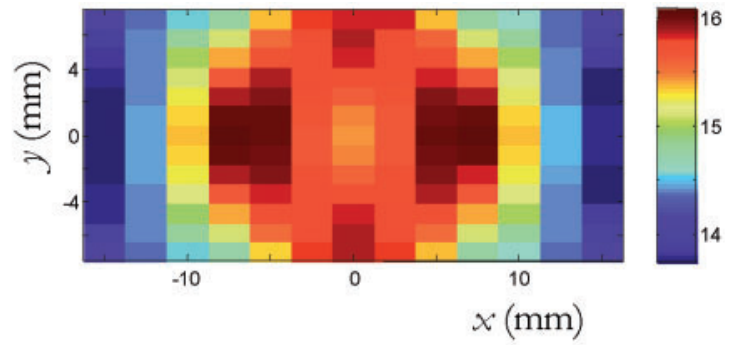

(b) deformation energy density $w_{d f}(\mathrm{MPa})$

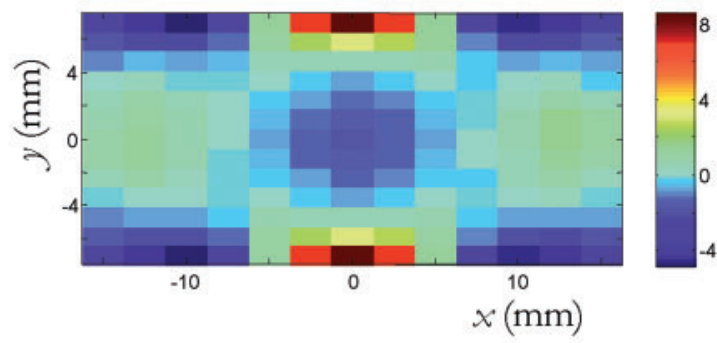

(d) relative uncertainty on the deformation

energy density $w_{d f}(\%)$

Fig. 6 Identified mechanical fields

was a velocity-controlled tensile loading up to a macroscopic strain of around 1 per cent. Then, the sample was completely unloaded before removing its two triangular-shaped sides by careful machining. The sample was then set again between the grips of the testing machine. It should be remembered here that these operations were not mandatory to estimate the deformation energy patterns. Their only purpose was to obtain a relatively well-known heterogeneous initial state. Images of the deforming specimen were taken by the digital camera during all the different mechanical loading stages. In order to minimize the error sources due to mounting and unmounting the specimen, rigid joints were used between the grips and the testing machine.

By comparing an image of the specimen in its reference state and after machining, the residual strain field could be estimated. Figure 7 shows the measured and simulated equivalent plastic strain at the end of the third load step. Although the actual behaviour of the material was surely different from the one used in the simulation, it can be observed that the measured and simulated residual plastic strain patterns matched rather well. As this plastic strain field was directly related to the residual stress state, it was thus assumed that the residual stress pattern developed within the sample was similar to the one exhibited in the numerical simulations.

\subsubsection{Experimental results}

The macroscopic stress-strain response of the sample during the fourth load step is given in Fig. 8. It shows the three load levels used for the identification (one in

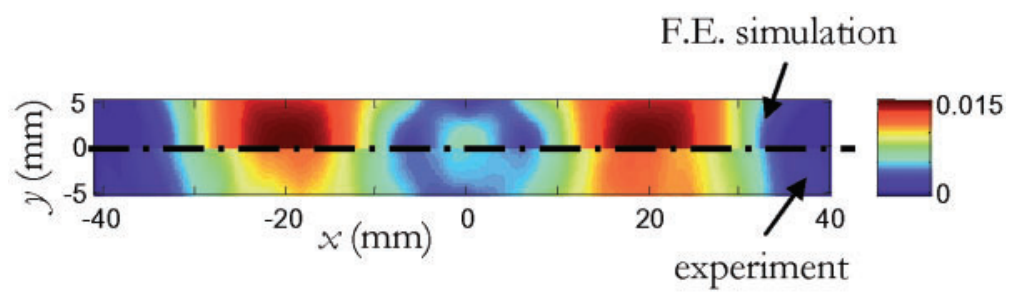

Fig. 7 Measured and simulated equivalent plastic strain at the end of the third load step 


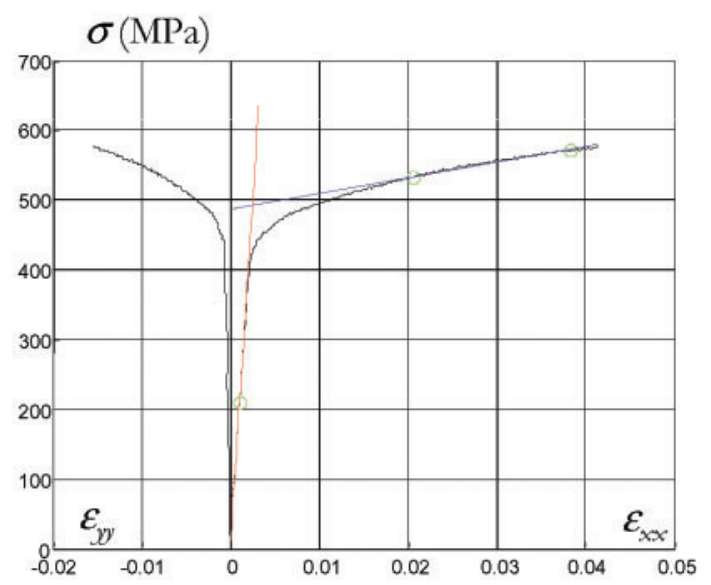

Fig. 8 Macroscopic stress-strain curve associated with the fourth load step

the elastic domain to identify the elastic parameters $E$ and $v$, and two in the plastic domain to identify the two plastic parameters $k$ and $\sigma_{0}$ ). Naturally, the material response did not perfectly match the theoretical one associated with a linear kinematic hardening model. Nevertheless, it was possible to estimate the 'macroscopic' homogeneous parameters corresponding to this material behaviour, using this global response. Naturally, the plastic parameters identified in this manner did not account for the heterogeneity of the initial state. They are given here uniquely to illustrate the order of magnitude of the material parameters: $E^{\text {macro }}=194 \mathrm{GPa}, v^{\text {macro }}=0.3, k^{\text {macro }}=$ $2.4 \mathrm{GPa}, \sigma_{0}{ }^{\text {macro }}=480 \mathrm{MPa}$.

Only the displacement fields measured at the three load levels represented in Fig. 8 were used for the material identification. They were computed on a regular virtual grid of $626 \times 82$ measurement points, using correlation subsets of $40 \times 40$ pixels and a biquadratic shape function for the subset transformation (as described in section 2).

The deformation field represented in Fig. 9 was computed using a sweeping local polynomial approximation method involving biquadratic approximation functions of the displacement field (which

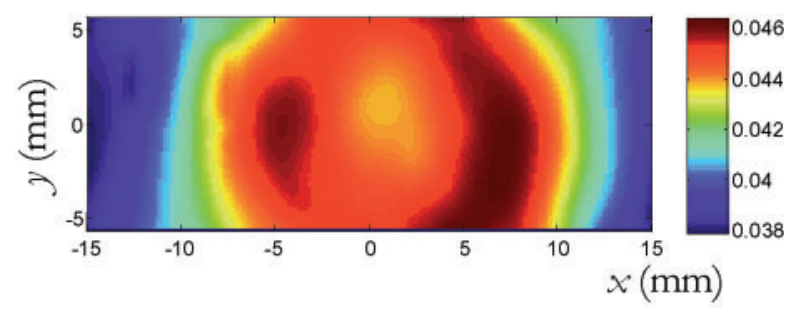

Fig. 9 Axial strain distribution $\varepsilon_{x x}$ at the end of the fourth load step was consistent with the supposed subset local transformation). This procedure also allowed recovery of the fitted displacement data. The difference between the measured and the filtered axial displacement in the central part of the sample is plotted in Fig. 10. It shows that the data fitting suppressed a random Gaussian error component associated with a zero pixel mean and a 0.02 pixel standard deviation (see Fig. 10(b)), without introducing bias on lowfrequency gradients.

\subsubsection{Deformation energy estimate}

The filtered displacement data were then interpolated on a coarser $13 \times 13$ grid before being used in the identification procedure in order to regularize the identification problem (see Fig. 11). They illustrate the symmetry of the measurements, which was consistent with the expected symmetry of the response of the specimen. From these displacement data the following elastic parameters were identified: $E^{\text {ident }}=192 \mathrm{GPa}$ and $v^{\text {ident }}=0.26$. They were close to the values deduced from the global stress-strain curve. These results also confirm the hypothesis of a non-damageable elasticity.

The plastic parameter distribution provided by the identification procedure is shown in Fig. 12. Their mean values were consistent with the ones derived from the analysis of the macroscopic stress-strain response of the sample: $k_{\text {mean }}^{\text {ident }}=1950 \mathrm{MPa}$ and $\sigma_{0 \text { mean }}^{\text {ident }}=480 \mathrm{MPa}$. As observed in the numerical results, the distribution of the identified yield stress $\sigma_{0}$ was quite symmetric with respect to both vertical and horizontal axes. Furthermore, it matched rather well the distribution associated with the numerical results. It can clearly be observed that the four corners of the domain corresponded to higher values of yield stress and that the horizontal free boundaries, located in the direct neighbourhood of the removed part of the sample, had the smallest level of yield stress. In addition, the vertical boundaries showed high identified yield stress values, while the yield stress identified in the middle of the domain was slightly larger than its mean value.

Conversely, distribution of the identified hardening modulus $k$ was slightly heterogeneous. The standard deviation on this parameter was around $120 \mathrm{MPa}$, which corresponds to approximately 6 per cent of $k_{\text {mean }}^{\text {ident }}$. This last result is also in good agreement with the expectation of a homogeneous distribution of this parameter.

The associated axial stress and strain distributions are plotted in Fig. 13. The patterns observed were 


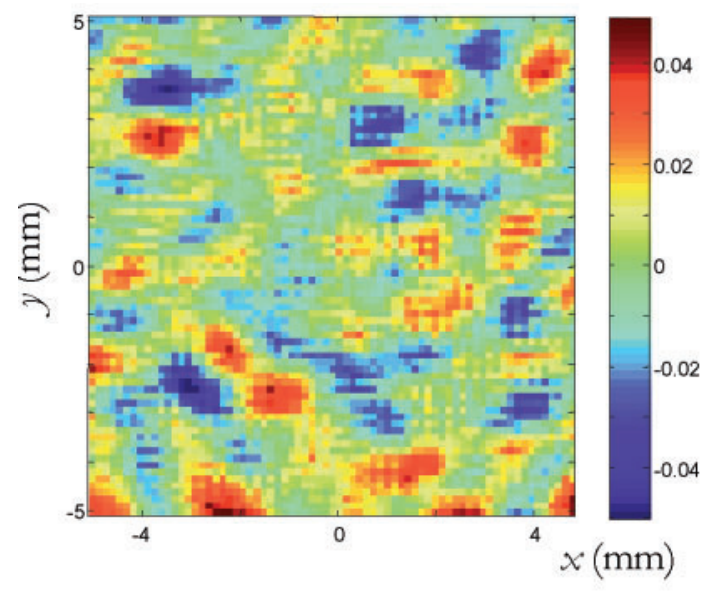

(a) $u_{x}-u_{x}^{\text {fit }}$ (pix.) for the $4^{\text {th }}$ load step

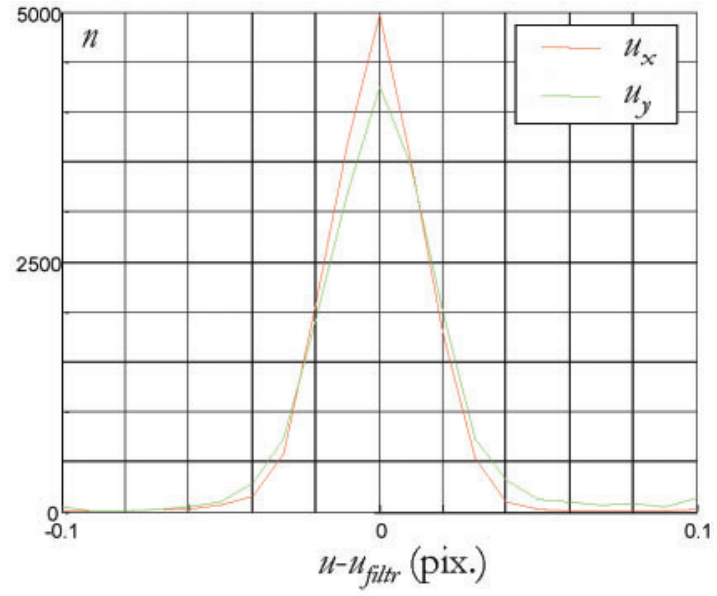

(b) histogram of $u-v^{\text {fit }}$ (pix.) for the $4^{\text {th }}$ load

step

Fig. 10 Residual on the displacement field after the fitting operation
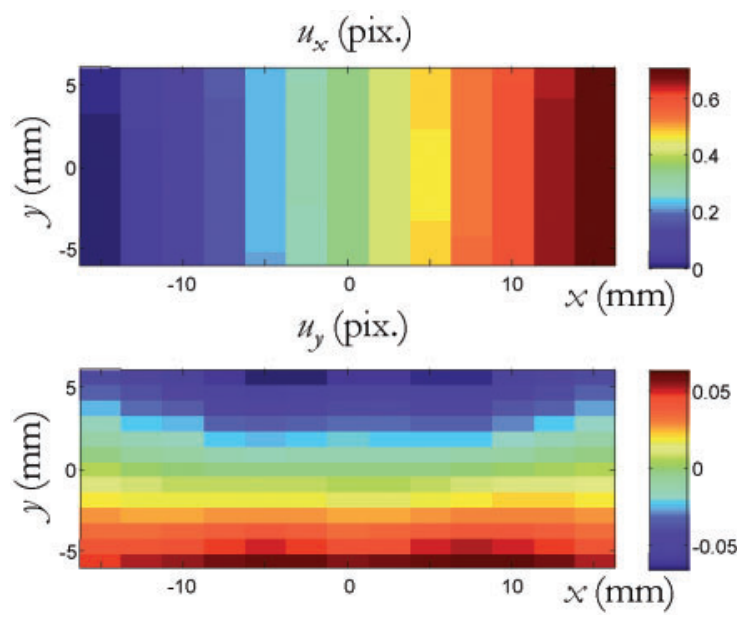

Fig. 11 Interpolated displacement field at the end of the fourth load step

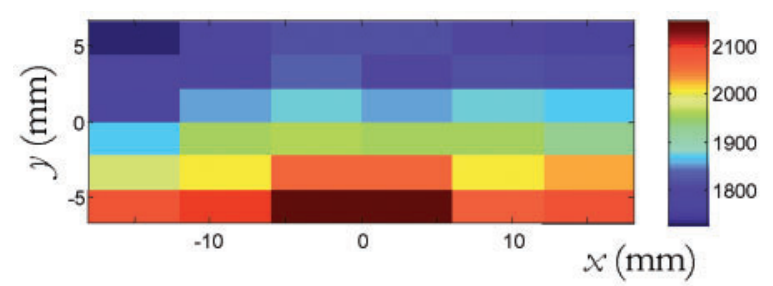

(a) $k^{\text {ident }}(\mathrm{MPa})$ also in good agreement with the numerical simulations presented in the previous section. Naturally, the stress amplitudes were slightly different from the ones numerically obtained because the materials hardening moduli were different: the highest stress levels were observed in the four corners of the domain and a stress concentration developed in the centre of the domain. Figure 13(b) shows that the axial strain field associated with the displacement data used for the identification was fully consistent with the experimental and numerical deformation fields shown in Figs 7 and 9.

The deformation energy density field was computed using the method described in the previous section. The estimate of the deformation energy density fields is given in Fig. 14 and is also in good agreement with the results derived from numerical simulations (see Fig. 6(b)). In particular, the smallest levels of deformation energy were observed in the

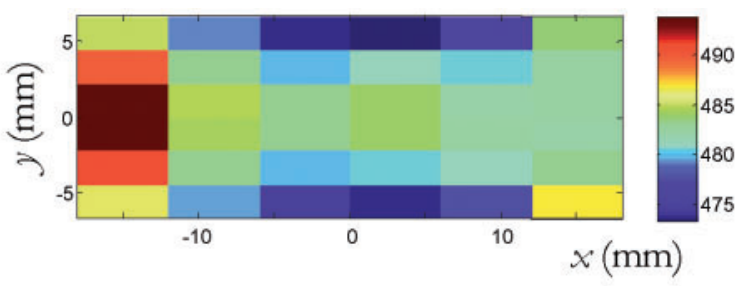

(b) $\sigma_{0}^{\text {ident }}(\mathrm{MPa})$

Fig. 12 Identified plastic parameters (hardening modulus $k$ and yield stress $\sigma_{0}$ ) 


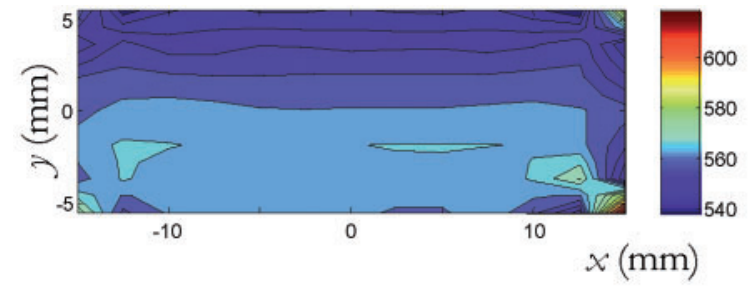

(a) $\sigma_{x \infty c}^{\text {ident }}(\mathrm{MPa})$

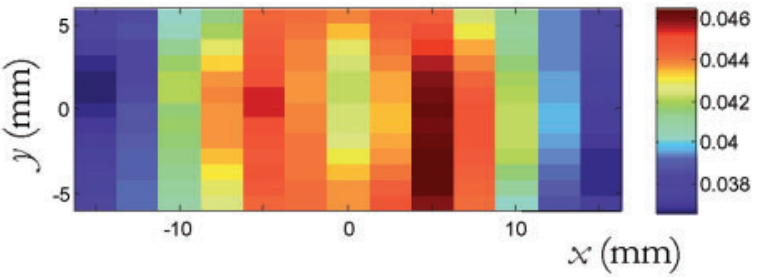

(b) $\varepsilon_{s c c}^{\text {ident }}$

Fig. 13 Identified axial stress component and axial strain used in the identification procedure

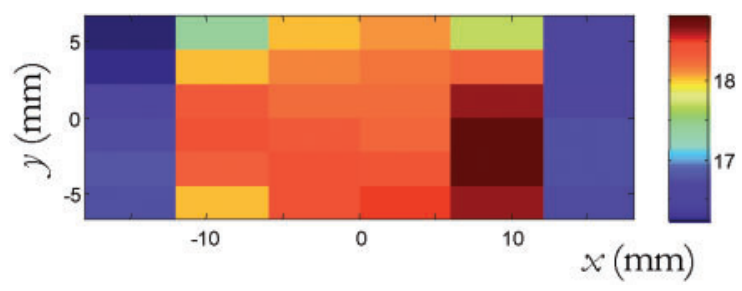

Fig. 14 Deformation energy density $w_{\text {def }}$ at the end of the fourth load step (MPa)

vertical boundaries of the domain, where the strains were minimal.

\section{CONCLUDING COMMENTS}

In conclusion, a procedure was designed allowing the measurement of deformation energy fields in specimens submitted to tensile mechanical loading. This procedure combines displacement measurements obtained by DIC with the stress fields given by a mechanical property identification method. Many technical difficulties were overcome and the first results presented in this paper are encouraging.

Mechanical property identification procedures from full field measurements are most generally developed to characterize homogeneous materials. In some situations (e.g. necking and fatigue), the response of a sample to simple loadings is not homogeneous, which can be interpreted as a heterogeneous material behaviour, associated with a heterogeneous initial state. For that reason, an identification procedure was devised that was capable of characterizing heterogeneous material behaviours. Inverse problems such as identification are known to be very sensitive to perturbations, being most likely noise in experimental data. Therefore, the method was first tested on numerical data obtained by a direct finite element computation. The mechanical hypotheses associated with the simulation were identical to the ones conceded in the identification procedure, small perturbations, plane stress, isotropic elasticity, and linear kinematic hardening, thus minimizing the errors due to the inconsistency between the chosen computational framework and the identification formulation. The error sources were then principally related to (a) inconsistent stress and strain gradient estimates due to the use of a coarser finite element mesh in the identification procedure than in the direct computation and (b) the imperfect hypothesis of piecewise constant material properties.

However, the material heterogeneity imposed in the numerical simulation was correctly retrieved by the developed procedure, and the corresponding deformation energy was also correctly estimated either in its pattern or its amplitude. These results emphasized the potential of the procedure.

The method was also applied to a set of experimental data associated with a heterogeneous material comparable with the one studied in the numerical test. The obtained results were also very encouraging: the trends in the identified material parameters (hardening modulus and yield stress) and in the deformation energy distributions matched the ones noticed in the reference numerical test. The observed discrepancies could be related to the difference between the 'real' material behaviour and its most academic modelling used here in the identification. The next step of this work will thus enlarge the panel of more realistic material models considered by the identification procedure. An important effort will also lead to improvement of the temporal integration of the deformation energy.

\section{REFERENCES}

1 Taylor, G. I. and Quinney, H. The latent energy remaining in a metal after cold working. Proc. $R$. Soc. Lond., 1934, A1 143, 307-326. 
2 Schmidt, A. O., Gilbert, W. W., and Boston, O. W. A thermal balance method and mechanical investigation for evaluating machinability. Trans. ASME, 1945, 67, 225-232.

3 Chrysochoos, A. Energy balance for elastoplastic transformation at finite strain. J. Mech. Théo. et Appl., 1985, V4(5), 589-614.

4 Chrysochoos, A. and Germain, M. Tensile test microcalorimetry for thermomechanical behaviour law analysis. Mater. Sci. Engng, 1989, A108, 25-32.

5 Rittel, D. On the conversion of plastic work to heat during high strain rate deformation of glassy polymers. Mechanics Mater., 1999, 31, 131-139.

6 Shenogin, S. V., Hohne, G. W., and Oleinik, E. F. Thermodynamics of the pre-yield deformation of glassy polymers: measurements with new deformation calorimeter. Thermochimica Acta, 2002, 391, 13-23.

7 Zehnder, A. T., Babinsky, E., and Palmer, T. Hybrid method for determining the fraction of plastic work converted to heat. Expl Mechanics, 1998, 38(4), 295-302.

8 Guduru, P. R., Zehnder, A. T., Rosakis, A. J., and Ravichandran, G. Dynamic full field measurements of crack tip temperatures. Engng Fract. Mechanics, 2001, 68, 1535-1556.

9 Chrysochoos, A. and Louche, H. An infrared image processing to analyse the calorific effects accompanying strain localisation. Int. J. Engng Sci., 2000, 38, 1759-1788.

10 Chu, T. C., Ranson, W. F., Sutton, M. A., and Peters, W. H. Application of digital-image-correlation techniques to experimental mechanics. Expl Mechanics, 1985, 25, 232-244.

11 Wattrisse, B., Chrysochoos, A., Muracciole, J.-M., and Némoz-Gaillard, M. Analysis of strain localization during tensile tests by digital image correlation. Expl Mechanics, 2001, 41(1), 29-39.

12 Doumalin, P., Bornert, M., and Caldemaison, D. Microextensometry by image correlation applied to micromechanical studies using the scanning electron microscopy. In Proceedings of the International Conference on Advanced technology in experimental mechanics, Japan Society of experimental Engineering, Ube City, Japan, 1999, pp. 81-86.
13 Lu, H. and Cary, P. D. Deformation measurements by digital image correlation: implementation of a second-order displacement gradient. Expl $\mathrm{Me}$ chanics, 2000, 40(4), 393-400.

14 Oulamara, A., Tribillon, G., and Duvernoy, J. Subpixel speckle displacement measurement using a digital processing technique. J. Mod. Opt., 1988, 35, 1201-1211.

15 Meuwissen, M. H. H., Oomens, C. W. J., Baaijens, F. P. T., Petterson, R., and Janssen, J. D. Determination of the elasto-plastic properties of aluminium using a mixed numerical-experimental method, J. Mater Processing. Technol., 1998, 75, 204-211.

16 Grédiac, M. and Pierron, F. Applying the virtual fields method to the identification of elasto-plastic constitutive parameters. Int. J. Plasticity, 2006, 22, 602-627.

17 Roux, S., Hild, F., and Pagano, S. A stress scale in full-field identification procedures: a diffuse stress gauge. Eur. J. Mechanics, A Solids, 2005, 24, 442-451.

18 Geymonat, G. and Pagano, S. Identification of mechanical properties by displacement field measurement: a variational approach. Meccanica, 2003, 38, 535-545.

19 Chrysochoos, A., Berthel, B., Latourte, F., Galtier, A., Pagano, S., and Wattrisse, B. Local energy analyses of HCF fatigue using DIC and IRT. J. Strain Analysis, 2008, 43(6), 411-422.

20 Latourte, F., Chrysochoos, A., Pagano, S., and Wattrisse, B. Elastoplastic behavior identification for heterogeneous loadings and materials. Expl Mechanics, 2008, 48(4), 435-449.

21 Simo, J. C. and Hughes, T. J. R. Computational inelasticity, 1998, pp. 126-130 (Springer, Berlin).

22 Geymonat, G., Hild, F., and Pagano, S. Identification of elastic parameters by displacement field measurement. C.R. Mécanique, 2002, 330, 403-408.

23 Wattrisse, B., Chrysochoos, A., Muracciole, J.-M., and Némoz-Gaillard, M. Kinematic manifestations of localisation phenomena in steels by digital image correlation. Eur. J. Mechanics, A Solids, 2001, 20, 189-211.

24 Batoz, J. L. and Dhatt, G. Modélisation des structures par éléments finis, 1990, vol. 1 (Hermès, Paris). 